\title{
Streaming SIMD Extensions
}

National Cancer Institute

\section{Source}

National Cancer Institute. Streaming SIMD Extensions. NCI Thesaurus. Code C64195.

A central controller which broadcasts an instruction stream to all the processing elements. 